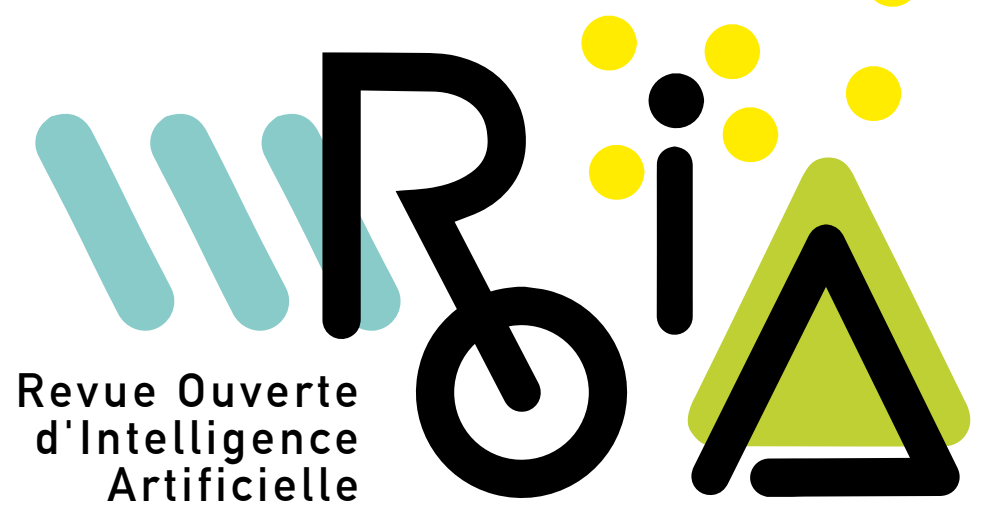

Frédérick Garçia, Thomas Guyet, Catherine Roussey Edito

Volume 2, no 1 (2021), p. 3-6.

<http://roia.centre-mersenne.org/item?id=ROIA_2021_2_1_3_0>

(C) Association pour la diffusion de la recherche francophone en intelligence artificielle et les auteurs, 2021, certains droits réservés.

\begin{tabular}{|l|l|l}
\hline (cc)BY & BY \\
\hline
\end{tabular}

Creative Commons Attribution 4.0 International License.

http://creativecommons.org/licenses/by/4.0/ 


\section{Revue Ouverte d'Intelligence Artificielle}

Volume 2, no 1, 2021, 3-6

\section{Edito}

L'agriculture, et plus en amont les sciences agronomiques, sont actuellement révolutionnées par l'essor du numérique. La diffusion des données et des équipements numériques dans les champs, les élevages et l'environnement conduit à imaginer une ferme du futur dans laquelle les machines agricoles deviennent connectées entre elles et en interaction avec les exploitants. Les systèmes de machines sont ainsi capable de collecter de la donnée, de les agréger et de les traiter afin d'accompagner l'utilisateur dans ses prises de décision, voire de piloter automatiquement une partie de son fonctionnement. Les champs sont surveillés continuellement par satellite ou par drones, les capteurs dans le sol ou sur les animaux alimentent en continu les systèmes d'informations des agriculteurs. Ceux-ci sont capables de prendre des décisions à partir de données locales mais aussi en interrogeant des sources de données distantes (prévision météo, simulation et archives, tweets). La décision est ensuite appliquée en commandant des automates à distance (irrigation, robots de traite, robots d'alimentation, etc.). La décision peut aussi être diffusée vers des systèmes distants comme le lancement des alertes sur les réseaux sociaux ou/et mobiles (risque de maladies, stade de développement des cultures, évènement climatique, etc.). Pour être efficace il faut que l'information transmise soit appropriée, que les bonnes décisions soient prises au bon moment dans un environnement pourtant complexe et évolutif.

Cette ferme numérique, évolution possible de l'agriculture de précision ou du smart farming déjà à l'œuvre aujourd'hui soulève des problèmes méthodologiques qui impliquent des données complexes, souvent volumineuses, hétérogènes et distribuées, parfois incomplètes et imprécises. Ces problèmes intègrent souvent une composante humaine prépondérante, multi-acteurs, dans un environnement évolutif impliquant des équipements de plus en plus autonomes. Toutes ces problématiques dessinent un terrain propice à la mise en place de méthodes de l'Intelligence Artificielle aptes à apporter des solutions originales, qui pourraient offrir des services nouveaux aux exploitants, aux futurs consommateurs et à l'environnement. Face à ces changements, le livre blanc Inrae/Inria [1] a mené un travail pour comprendre, maîtriser, préparer et accompagner le déploiement du numérique en agriculture au sein des exploitations et des filières au service des agriculteurs, de l'agriculture et du bien commun.

Ce numéro spécial de la revue ouverte d'Intelligence Artificielle (ROIA) propose une image représentative des recherches actuelles sur l'utilisation des techniques d'intelligence artificielle à l'agriculture. Nous avons sollicité des contributions montrant la pertinence, la faisabilité pratique et la largesse thématique de l'Intelligence Artificielle à la ferme numérique de demain. Ce numéro a ainsi été organisé en deux grandes parties. Les premiers articles traitent des approches d'analyse de données par des méthodes d'apprentissage automatiques. Les données traitées sont des images issues de différents capteurs. Nous avons ensuite regroupé les articles qui exploitent des méthodes de modélisation des connaissances et des acteurs pour mieux comprendre le fonctionnement complexe des exploitations. 
L'article de Rémi Régnier et al. présente de manière originale le bilan du challenge ROSE, première compétition mondiale de robotique et d'Intelligence Artificielle visant à évaluer les performances de robots de désherbage en conditions réelles sur des parcelles de cultures. Avec ce challenge, les auteurs abordent ainsi la question générale de la caractérisation de systèmes robotiques intelligents. Avec ce travail, les auteurs posent ainsi les bases de futures méthodologies et protocoles harmonisés d'évaluation des futurs systèmes de robotique agricole.

Les deux articles suivant proposent des méthodes d'apprentissage en analyse d'images pour évaluer les rendements agricoles. L'article suivant, intitulé « Traitement d'images et apprentissage automatique pour la viticulture de précision », est une collaboration menée au sein du projet européen H2020 «Artificial Intelligence for Digitizing Industry ». Cet article présente un état de l'art sur les différentes techniques d'analyse d'images de la vigne pour prédire les rendements. La prédiction du rendement peut se faire à différents stades de développement de la vigne. Dans ce cas les éléments à détecter varient : inflorescences, fleurs, grappes, baies. Cet article présente les techniques classiques d'apprentissage automatique et d'apprentissage profond utilisées par types d'éléments à détecter. Les résultats de ces techniques sont comparés en termes de nombre d'images, types d'images, types de prise de vues, cépages, métriques utilisées, paramétrage. Les auteurs concluent que les techniques classiques nécessitent un paramétrage manuel complexe contrairement aux techniques d'apprentissages profonds. Malheureusement celles-ci sont gourmandes en termes de quantités de données étiquetées manuellement et en puissance de calcul rendant les techniques classiques beaucoup plus abordables. Le besoin d'un benchmark contenant une grande quantité d'images hétérogènes se fait sentir pour pouvoir comparer efficacement toutes ces techniques. Cet état de l'art montre que même au-delà des aspects techniques informatiques, les conditions de prise de vue et les médias sont très variables et ont un impact important sur les résultats.

Le troisième article, d'Eliott Jacopin et al., porte sur la détection et le comptage automatique de plants sur des parcelles de grande culture, à partir d'images aériennes. Une telle méthode de détection permettrait aux agriculteurs de mieux prédire l'état de la culture et son futur rendement, et offrirait aux agronomes un moyen simple et rapide d'acquérir des données expérimentales. La méthode proposée repose d'une part sur un apprentissage non supervisé à base de clustering pour une première identification des rangs et des plantes, puis sur l'utilisation d'un système multi-agents nécessitant peu de paramètres pour affiner cette estimation et bien isoler chaque plant. Cette association d'apprentissage et de résolution par SMA est originale et permet d'obtenir expérimentalement de très bons résultats.

Les techniques d'Intelligence Artificielle peuvent aussi aider à la planification des cultures.

Dans l'article de Maqrot, de Givry, Tchamitchian et Quesnel intitulé «Conception de systèmes de verger-maraîcher à l'aide de la programmation mathématique », les auteurs proposent de résoudre la planification de cinq cultures maraîchères (salade, 
tomate, oignon, melon et carotte) dans une parcelle agroforestière contenant des pommiers. Tout d'abord, les auteurs montrent la difficulté de choisir l'emplacement des arbres et des cultures sur une même parcelle du fait de leurs interactions. Les arbres procurent de l'ombre aux cultures et limitent les mouvements de l'air influençant ainsi le stress thermique et d'évaporation des cultures. Ces interactions sont dites aériennes. Les systèmes racinaires des arbres et les cultures sont en compétition pour récupérer l'eau des sols. Ces interactions sont dites souterraines. De cette description générique les auteurs ont choisi de modéliser les interactions aériennes et souterraines pour formaliser sous forme mathématique du problème de planification des cultures sur deux ans consécutives dans une même parcelle. Ces travaux proposent deux modèles mathématiques pour résoudre la planification des cultures : Un modèle quadratique en variables binaires, un modèle linéaire en variables mixtes. Ces deux modèles ont été implémentés sur deux logiciels d'optimisation Cplex et LocalSolver. Les solutions préliminaires ont été obtenues en un temps raisonnable, malgré le nombre important de variables. Toutefois, ces solutions devront être améliorées car, contrairement à la pratique des agriculteurs, elles scindent la parcelle en deux : une zone mixte arbre-culture et une zone de cultures maraîchères.

Les deux derniers articles s'intéressent à des outils d'Intelligence Artificielle variés pour simuler et analyser des systèmes complexes, tels que ceux rencontrés dans des applications agro-environnementales.

Dans l'article intitulé «simulation du passage des intentions aux actions en agriculture », R. Martin-Clouaire et J.-P. Rellier présentent un outil de modélisation de système production agricole orienté vers la planification d'actions. L'objectif de ce travail est de proposer un formalisme de modélisation qui soit adapté aux spécificités des systèmes complexes que sont les exploitations agricoles. En particulier, il s'agissait de prendre en compte les incertains au cours du temps (incertains climatiques, de production), et l'organisation du travail (contraintes de personnel et de ressources matérielles). Pour les auteurs, il s'agit alors de définir un formalisme de définition d'un plan flexible, c'est-à-dire d'un plan d'exécution qui sera opérationnellement dépendant du déroulé effectif des opérations en tenant compte des impondérables. Ce travail montre donc toute la complexité de l'organisation des exploitations agricoles et montre tout l'apport que peuvent avoir les méthodes de raisonnement automatiques (planification, raisonnement temporel) pour améliorer la gestion de celles-ci : pour leur efficience, pour faciliter le travail des exploitants et pour une utilisation plus raisonnée des ressources.

Dans l'article de L. Sadou et al., un modèle de dynamique de l'acceptation d'une innovation est analysé. Plus précisément, les auteurs s'intéressent à comprendre la dynamique de l'acceptation de la mise en place de compteurs d'eau connectés dans une région agricole. Ils s'appuient pour cela sur une simulation multi-agents fondée sur l'argumentation, afin de mieux comprendre sur quels leviers s'appuyer pour influencer les agriculteurs (les agents) à adopter des compteurs communicants. Ce travail se situe donc à l'interface entre les domaines de l'argumentation et de la simulation multi-agents. 
Nous espérons que ces travaux montreront l'impact de l'Intelligence Artificielle pour construire l'agriculture numérique, mais aussi qu'ils démystifieront ce que l'IA peut réellement faire et ne peut pas faire ...

\section{Bibliographie}

[1] V. Bellon-Maurel, L. Brossard, F. Garcia, N. Mitton, E. Prados \& A. Termier, «Livre blanc INRIA / INRAE sur le numérique en Agriculture », in PFIA 2020 - (Plateforme de l'Intelligence Artificielle) - Journée Agronomie et IA (Virtual, France), Jun 2020, p. 1-26. 